\title{
Aperçu sur l'évolution de la technique des vannes pour installations hydroélectriques
}

\section{A review of hydro-electric gate design trends}

\author{
PAR G. DAUMY, \\ CHEF DU DÉPARTEMENT HYDRAULIQUE ET CHARPENTE \\ DES ETTABLISSEMENTS BOUCHAYER \& VIALLET, GRENORLE
}

\begin{abstract}
L'évolution de la technique des vannes est normalement la conséquence des progrès réalisés dans le domaine de l'Indraulique théorique on dans le domaine général de la construction mécanique. Cette évolution concerne aussi bien les tabliers (généralisation des structures en coque, ...) que les appareils de manouvre (utilisation des treuils monoblocs standardisés,...) et se retrouve $\dot{a}$ tous les stades : conception, fabrication, montage. Les progrès techniques de la dernière décennie visent essentiellement l'économie, la sécurité et la facilité d'exploitation.
\end{abstract}

\begin{abstract}
Grate design trends are normally determined by developments in theoretical hydratics or in the field of general mechanical engineering. These denelopments affect both gate leaf design (the more general use of thin-shell structures, etc.) and control equipment (the use of siandard unit-construction hoists, etc.); their influence shows up at all stages, from the drawing board to final assembly. Fconomy and operational reliability and convenience have been the main features of technical progress achieved during the past ten years.
\end{abstract}

La technique et la technologie des vannes évoluent en permanence dans un sens favorable, à un prix de plus en plus réduit et à un service toujours plus sûr. Cette évolution est parfois provoquée par des besoins nouveaux qui surgissent; c'est le cas, par exemple, de certaines vannes pour usine marémotrice ou de vannes à usages multiples. Mais, plus généralement, l'évolution est fonction de la connaissance de plus en plus approfondie des phénomènes hydrauliques, ainsi que des progrès réalisés dans les techniques visant plus ou moins directement la construction mécanique.

La connaissance de plus en plus précise des phénomènes hydrauliques, et lout particulièrement des phénomènes hydrauliques locaux, influe directement sur l'implantation et la forme à donner aux organes de coupure que sont les vannes de types divers : vannes-segments, vannes-wagons, batardeaux, vannes-clapets, vannes à papillons, etc. Il importe en effet de réduire au minimum les sollicitations hydrauliques exercées sur les structures et la valeur des efforts nécessaires pour la manœuvre des vannes.

Les techniques, en cours de progrès rapide, apportent des modifications considérables dans le domaine des vannes, par les nouvelles possi- 


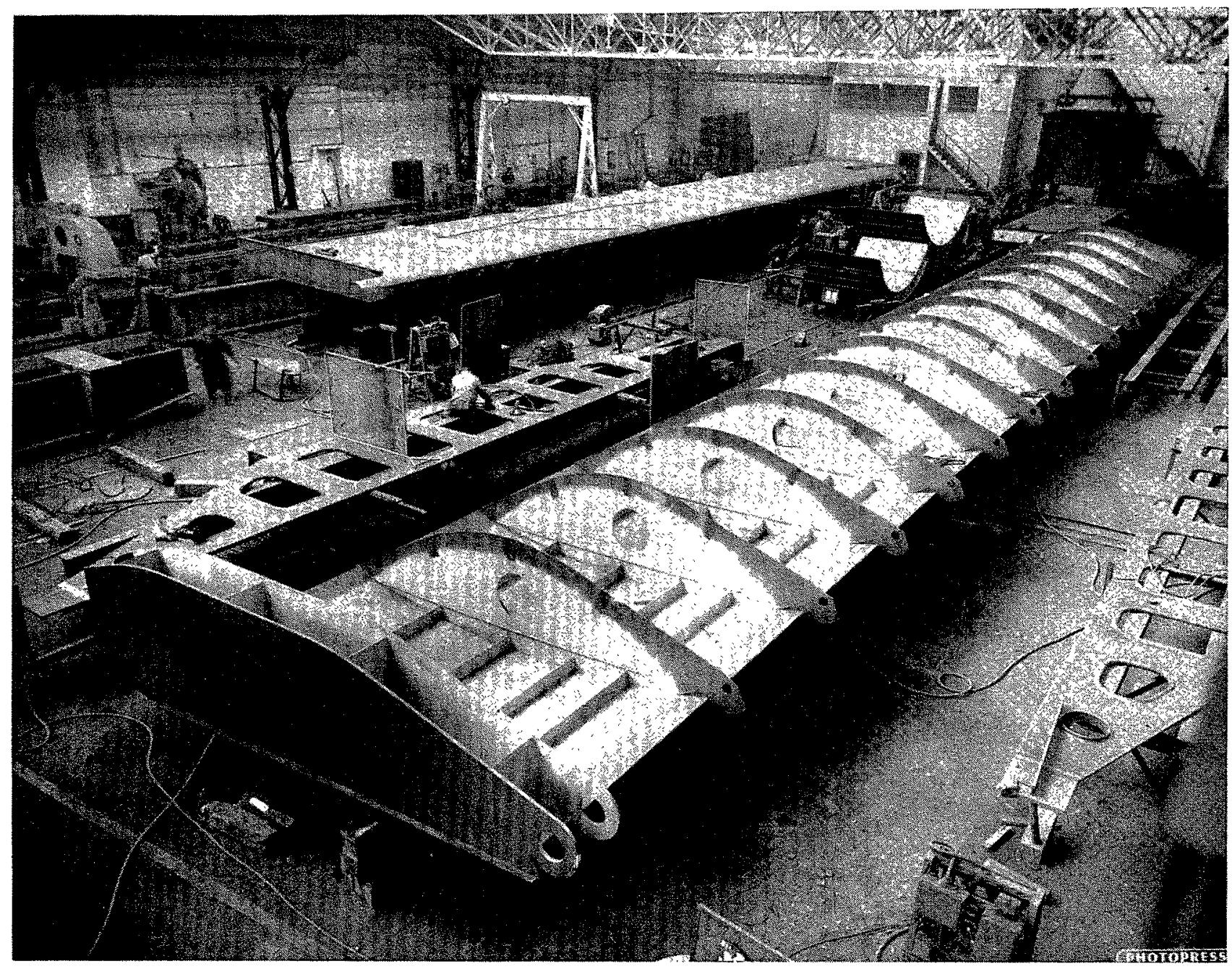

FIG. 1

(Cliché Ets Bouchayer \& Viallet.)

Une vanne-clapet, destinée au barrage de Vichy, en cours de fabrication. - Longueur: $29,50 \mathrm{~m}$; Hauteur utile: $4,10 \mathrm{~m}$. Structure en coque. Le tablier de la vanne repose sur son bordé. On distingue trés nettement les diaphragmes transversaux et les lisses longitudinales de raidissement. La tôle de contre-bordé sera mise en place par la suite.

bilités qu'elles offrent aux fabricants et par le déplacement de ce que l'on peut appeler le barycentre économique de la construction. L'économie et la sécurité maximales sont en effet le résultat d'un compromis entre diverses tendances souvent contradictoires, visant les matières premières, la main-d'œuvre de fabrication en atelier, la facilité de transport et de montage sur chantier, etc.

Un nouveau procédé de soudure sur chantier peut remettre en cause la conception même d'une vanne, et par voie de conséquence, le choix des aciers de construction et les procédés de fabrication. C'est ainsi que la conception des grandes vannes évolue vers une fabrication d'éléments constitutifs de plus en plus lourds, assemblés sur chantier par soudure, au lieu de couvre-joints rivés.

La généralisation des structures en coque, avec bordé et contre-bordé soudés, raidis par lisses et solidarisés par voiles transversaux, est dérivée directement des techniques de construction aéronautique. Elle est particulièrement fructueuse dans le cas des clapets, des vannessecteurs ou des vannes-wagons de grandes dimensions.

Par ailleurs, de nouvelles possibilités sont en permanence proposées aux constructeurs :

- nouvelles nuances d'acier inoxydable, répondant mieux aux besoins; 
Fig. 2

Eléments de vannes-wagons, destinées à l'équipement marémoteur de la Rance, en cours de fabrication. Structure de tablier en coque largement ajourée. Largeur : $15 \mathrm{~m}$. Chaque vanne comporte six éléments analogues superposés (découpage vertical) qui sont assem.

blés au montage sur place.

(Cliché Ets Bouchayer
\& Yiallet.)
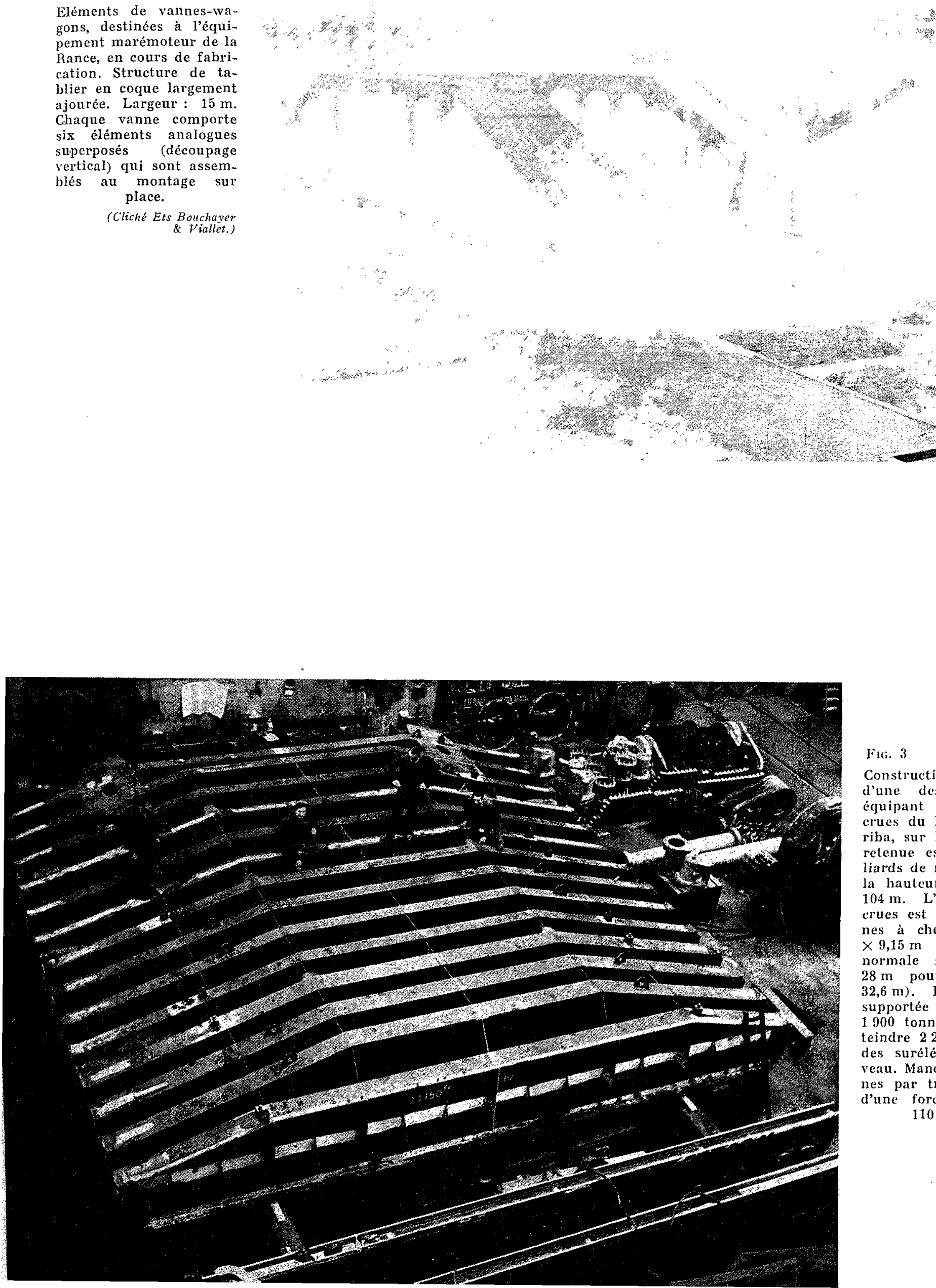

Fici. 3

Construction en atelier d'une des six vannes équipant l'évacuateur' de crues du barrage de Kariba, sur le Zambèze. La retenue est de 160 milliards de mètres cubes et la hauteur de chute, de $104 \mathrm{~m}$. L'évacuateur de crues est équipé de vannes a chenilles de 8,85 $\times 9,15 \mathrm{~m}$ (charge d'eau normale sur le seuil : $28 \mathrm{~m}$ pouvant atteindre $32,6 \mathrm{~m})$. Poussée totale supportée par une vanne: 1900 tonnes pouvant atteindre 2280 tonnes lors des surélévations de niveau. Mancuvre des vannes par treuils à câbles d'une foree unitaire de 110 tonnes.

(Cliché Neyrpic.) 


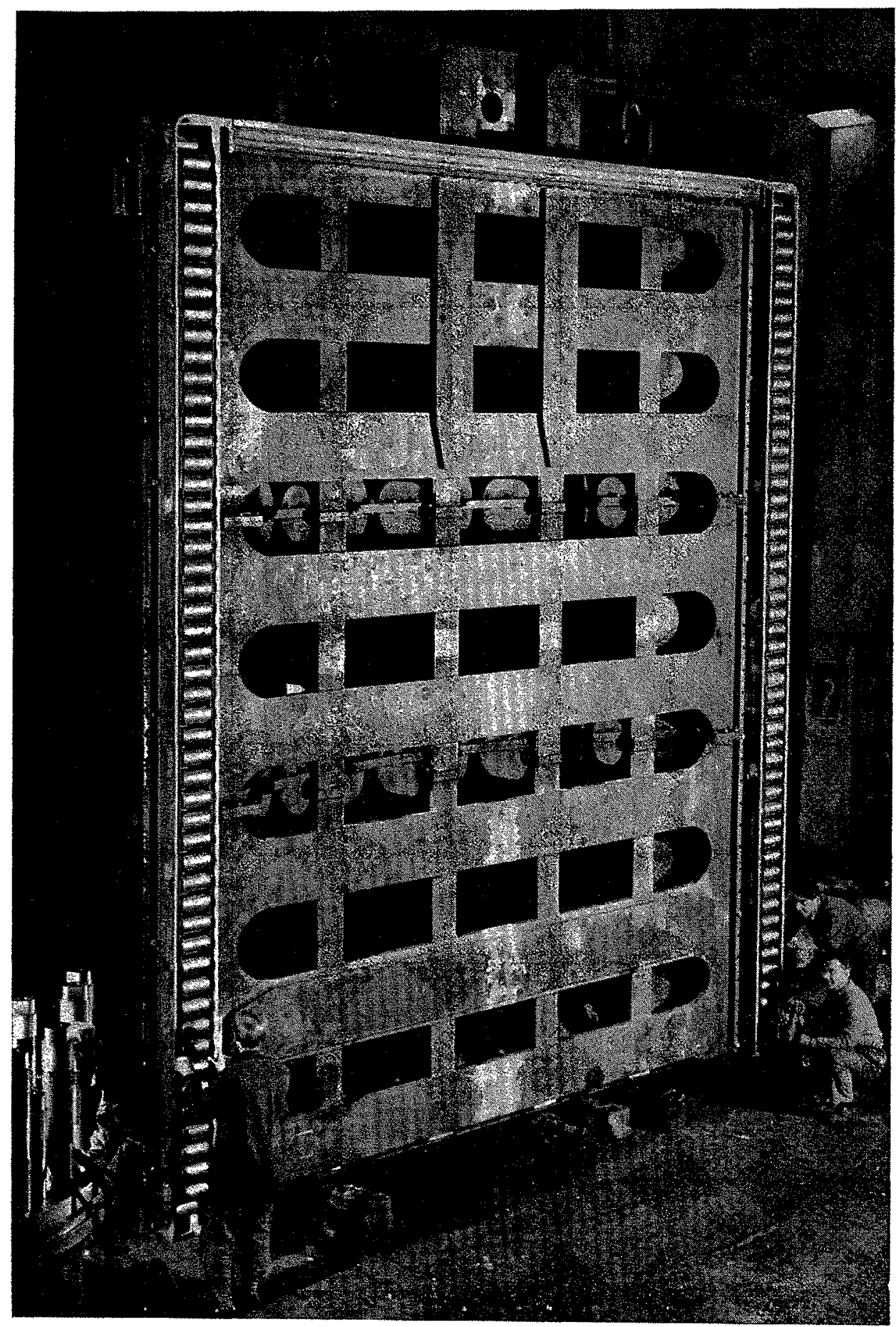

(Cliché Neyrapic.)

FIG. 4

Vue en atelier d'une des tróis vames de prise d'eau équipant le barrage de Valdecanas sur le Tage. Vannes à chenilles de $4,8 \times 5,8 \mathrm{~m}$. Charge d'eau sur le seuil : $87,9 \mathrm{~m}$; poussée totale sur une vanne : 2300 tomes. Manouvre des vannes par servo-moteurs à huile sous pression. 


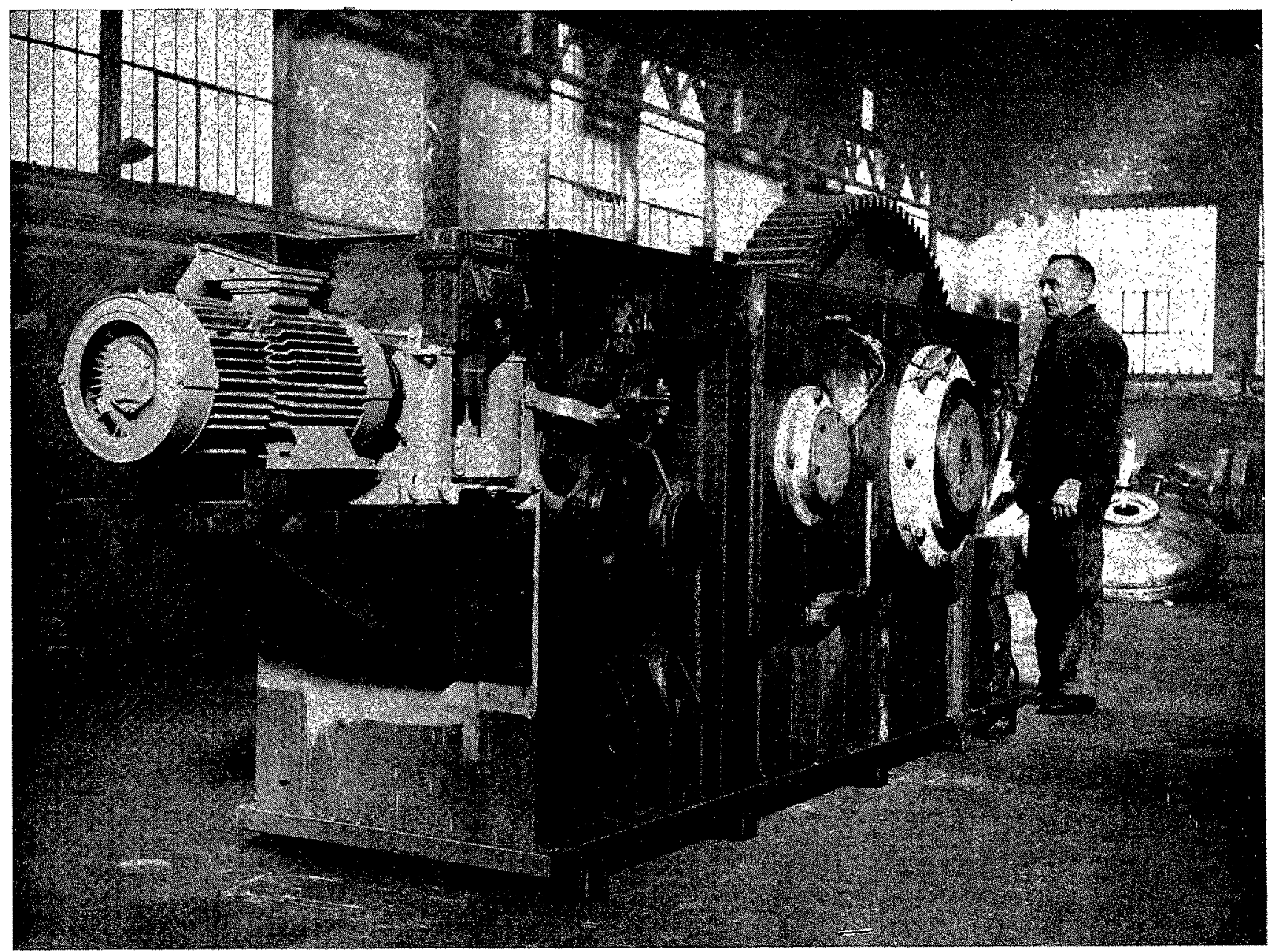

(Cliché Ets Botchayer d Viallet.)

FiG. 5

Treuil monobloc standard pour levage de vanne par chaine Galle. Force: 100 tonnes. Le capot supérieur a été enlevé. Le treuil est entièrement équipé avec noteur, appareillage de commande et de sécurité.

- nouveaux thermoplastiques à haute résistance, de plus grande stabilité dimensionnelle et à bas coefficient de frottement : téflon, delrin, rilsan;

- nouveaux caoutchoucs synthétiques plus durables et plus résistants;

- extension des possibilités de traitement des surfaces, tels que bronzage ou chromage dur en paliers ou sur tourillons de plus en plus gros; etc.

La sécurité quasi absolue acquise dans certains types de servocommandes oléohydrauliques ou d'appareillages électriques, conduit à en généraliser l'emploi et à introduire ces genres de matériels dans des installations assez rustiques et isolées, ou jadis ils eussent été proserits.

Il est maintenant courant de remplacer la synchronisation mécanique de treuils accouplés pour le levage d'une vanne par une synchronisation purement ćlectrique.

Les treuils pour mancuvre des vannes par câble ou par chaine tendent à être slandardisés, et ceci malgré la très grande diversilé des cas qui se présentent, puisque chaque installation possède ses propres caractéristiques : hauteur de chute, débit, largeur possible, ete. Des treuils monoblocs standards, adaptés aux efrorts et aux vitesses courants, permettent d'installer une mécanique de haute qualité parce qu'étudiée 


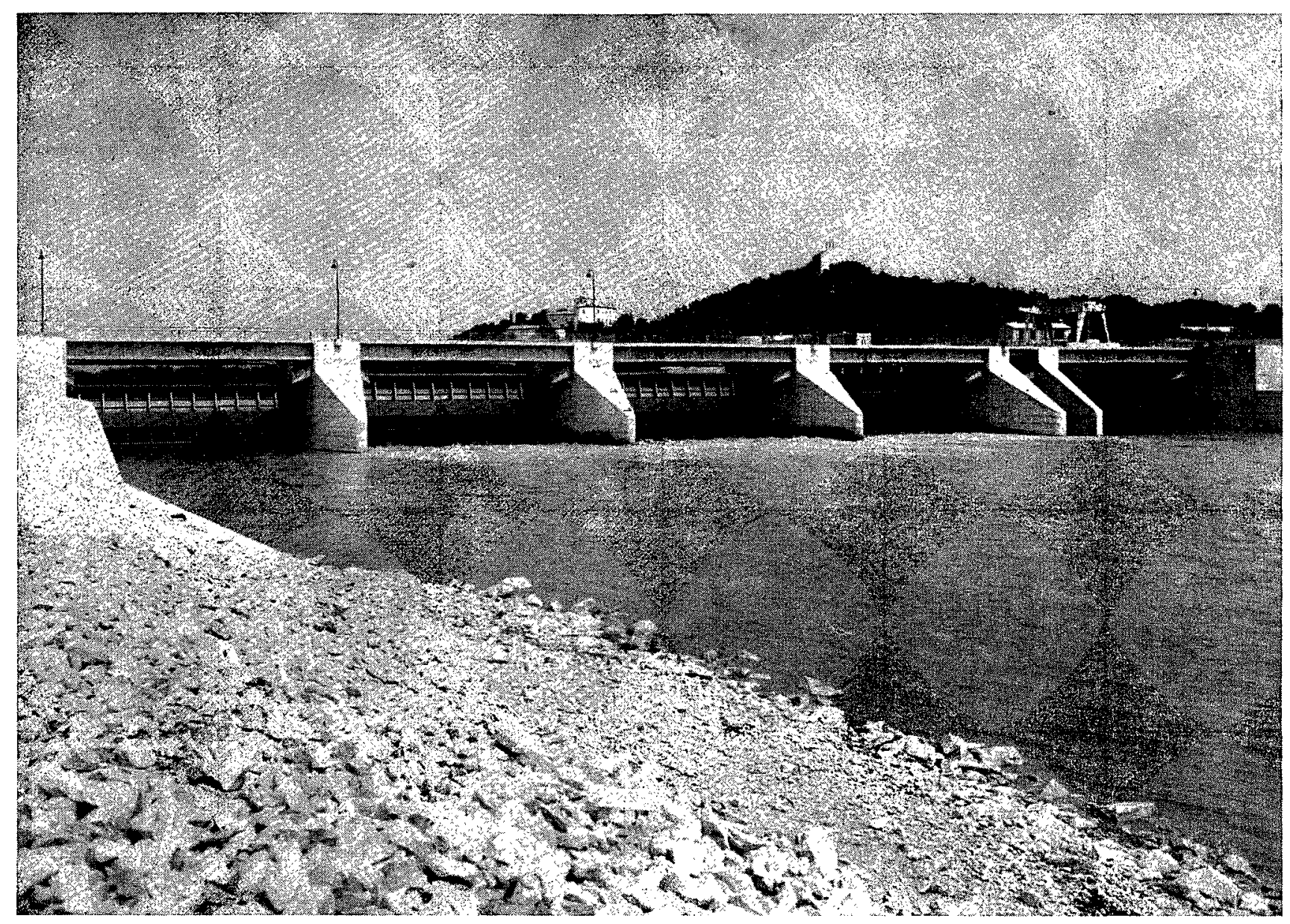

(Cliché S.F.A.C.)

FIG. 8

Barrage de Cadarache sur la Durance.

Les cinq vannes-segment $\mathrm{du}$ barrage mobile : $25 \times 6,50 \mathrm{~m}$.

pour un grand nombre d'exemplaires, avec un encombrement très réduit et au prix minimal. En outre, de tels treuils à graissage automatique et capotage parfait sont susceptibles d'être disposés en plein air sans nécessité de cabine de manœuvre.

Les progrès réalisés dans le domaine des vannes proprement dit, sont d'autre part, susceptibles de provoquer des économies de génie civil. C'est ainsi que l'utilisation de treuils monoblocs à faible encombrement permet de réduire la largeur des piles séparant les pertuis, c'est-àdire le cubage de béton armé de l'installation.

Au cours de ces dernières années, les caracléristiques des vannes n'ont pas connu d'augmentation spectaculaire. Les grandes vannes des barrages de navigation dépassent ravement les gabarits classiques de 30 ou $46 \mathrm{~m}$ de largeur. De même, la poussée de 8000 à $10000 \mathrm{t}$ pour lea vannes de fond les plus chargées n'est guc̀re dépassée. Par ailleurs, la vanne-segment évacuateur de crue disposée en crête de barrage est, dans la pratique, limitée à $16 \times 16 \mathrm{~m}$. Il esl à noter cependant une certaine tendance à évacuer tout ou partic des crues par des vannes en charge, et les constructeurs de barrage proposent de plus en plus souvent des vannes-secteurs importantes sous très forte charge : 45,60 et même $120 \mathrm{~m}$.

En tout cas, la course aux records, pour autant qu'elle existe, est lente. Cela semble tenir au fait que, contrairement au cas des transports aériens, l'optimum économique ne lui est pas directement lié. A l'heure actuelle, le progries technique dans le domaine des vannes se traduit essentiellement par l'économie, la sécurité de l'installation et la facilité d'exploitation. 